\title{
Homogénéisation numérique de la résistance mécanique des massifs fracturés
}

A. POUYA

M. GHOREYCHI

G.3S, École polytechnique 91128 Palaiseau Cedex pouya@g3s.

polytechnique.fr mg@g3s.polytechnique.fr
Une méthode pour déterminer les propriétés de résistance des massifs rocheux fracturés est proposée, à partir de I'homogénéisation numérique par la méthode des éléments finis. Les différentes familles de fractures sont générées à partir des données disponibles et des lois statistiques. Le comportement mécanique de chaque fracture est modélisê en élastoplasticité, en introduisant les caractéristiques mesurées au laboratoire ou in situ: les raideurs normale et tangentielle, la cohésion, l'angle de frottement interne et la dilatance. Le comportement de la matrice est également modélisé en élastoplasticité. Après la détermination numérique du Volume Élémentaire Représentatif, plusieurs simulations sont effectuées avec différents trajets de charge. Leurs résultats révèlent qu'au-delà du VER, le comportement global du massif fracturé est élastoplastique avec écrouissage. Il est caractérisé par une surface de charge orientée dont l'expression est donnée. Les valeurs des résistances anisotropes sont également déterminées.

Mots-clés : massifs rocheux, milieux fracturés, comportement mécanique, critère de résistance, élastoplasticité, homogénéisation.
NDLE: Les discussions sur cet article sont acceptées jusqu'au 1" octobre 2001.
A method for determining strength properties of fractured rock masses is proposed. It is based on numerical homogeneisation using finite element method. Different farnilles of fractures are generated on the basis of data available and statistical laws.

Mechanical behaviour of each fracture is modelled in elastoplasticity by introducing properties measured in laboratory or in situ : normal and tangential stiffnesses, cohesion, angle of internal friction and dilatancy. Matrix behaviour is also modelled in elastoplasticity. After determination of Representative Elementary Volume, several simulations are performed with different loading paths. The results show that beyond REV, giobal behaviour of fractured rock mass is elasoplastic with hardening. It is characterised by an oriented criterion the expression of which is given. The values of anisotropic strength are also determined.

Key words : rock mass, fracture, strength criterion, mechanical behaviour, elastoplasticity, homogenization. 


\section{Introduction}

On considère un massif fracturé dont les propriétés mécaniques peuvent être supposées homogènes à une échelle suffisamment grande. A cette échelle, le comportement du massif peut être représenté par celui d'un milieu continu homogène équivalent (MHE). Plusieurs méthodes ont été proposées dans la littérature pour la "classification des massifs fracturés ». Elles permettant de déterminer, d'une manière empiriquue et plutôt qualitative, les propriétés homogénéisées du massif, en fonction de celles de la matrice rocheuse et de certaines caractéristiques de la fracturation (Barton et al., 1974; Bieniawski, 1976). Lorsqu'une ou deux directions préférentielles de la facturation sont considérées, et ceci engendre une périodicité dans la structure du milieu, certaines propriétés mécaniques du massif peuvent être calculées d'une manière rigoureuse et par des méthodes semi-analytiques, en fonction de propriétés de la matrice rocheuse et des fractures (Bekaert et Maghous, 1996 ; de Buhan et Maghous, 1997).

La démarche présentée ici se situe entre ces deux extrêmes : nous considérons le cas de massifs dont la fracturation est exprimée par des lois statistiques quelconques décrivant l'orientation, l'extension, la densité et l'épaisseur des fractures. Nous tentons de déterminer les propriétés du MHE par la voie numérique. Nous étudions pour cela le comportement d'un domaine suffisamment grand du massif fracturé pouvant représenter le comportement moyen de ce massif. Le comportement de ce volume élémentaire représentatif (VER) est étudié par la méthode des éléments finnis, en assimilant les fractures à des éléments u joints ». Cette méthode a déjà été appliquée à l'étude des propriétés d'élasticité non linéaire du massif (Coste, 1997 ; Coste et al., 1999). Ce travail en constitue une extension et porte essentie]. lement sur la détermination de la résistance mécanique du milieu fracturé dont le comportement global est du type élastoplastique.

\section{2}

\section{Présentation de la méthode}

\section{2.}

\section{Description de la fracturation}

Le relevé géologique de la fracturation fournit des informations sur l'orientation (pendage et azimut), la lonqueur, l'espacement (densité de fractures par mètres de carotte) et l'épaisseur des fractures. Ces informations sont souvent très incomplètes. Elles permettent cependant, moyennant certaines hypothèses, de définir des familles de fractures qui peuvent être assimilées à des disques, dans un espace à trois dimensions, Cette hypothèse est fréquemment adoptée dans la modélisation de l'écoulement en milieu fracturé (Long et al., 1987) et peut être également admise pour une modélisation mécanique. Toutefois, nous allons nous restreindre ici à une modélisation plus simplifiée à deux dimensions. Nous sommes conscients de l'insuffisance de cette représentation dans le cas de milieux fracturés et plaçons cette première étude dans la perspective plus large d'une extension ultérieure à trois dimensions. Ce choix est motivé par l'objectif spécifique de cette étude qui consiste à déterminer la résistance mécanique du milieu fracturé. Cela exige le recours à de nombreux calculs en élastoplasticité, en présence de nombreuses fractures. Ce type de modélisation n'est pas encore tout à fait opérationnel à trois dimensions.

Aussi, dans cette étude, nous allons considérer que les différentes familles de disques sont recoupées par différents plans, ce qui permet d'en déduire des modèles de fractures à deux dimensions.

Dans l'exemple du massif étudié, les fractures ont une extension variant de $20 \mathrm{~cm}$ à $30 \mathrm{~m}$ suivant une loi de distribution statistique exponentielle. Leurs orientations varient entre 0 et $90^{\circ}$ par rapport à l'axe $\mathrm{x}$ du VER (Fig. 1) et suivent une loi normale avec une movenne d'environ $60^{\circ}$ (fractures sub-verticales) et un écart-type de $10^{\circ}$. La densité surfacique des fractures (nombre de centres de fractures par unité de surface) suit une distribution uniforme, avec une valeur moyenne d'environ $0,4 \mathrm{~m}^{-2}$ L'épaisseur des fractures est supposée de l'ordre de $1 \mathrm{~mm}$ (fractures essentiellement colmatées).

\section{2}

\section{Comportement de la matrice et des fractures}

Pour simplifier le modèle, nous considérons que le massif a un comportement mécanique élastoplastique du type Mohr-Coulomb. Ce type de comportement est fréquent en mécanique des roches et a servi en particulier aux autres approches citées ci-dessus.

On suppose par ailleurs que le comportement élastoplastique de la matrice rocheuse (sans fracture) est caractérisé par une élasticité linéaire et isotrope et une plasticité parfaite. On néglige ainsi la rupture fragile de la matrice qui n'intervient que pour des contraintes très élevées par rapport à la résistance au cisaillement des fractures. Le comportement des fractures assimilées à des « joints n est également linéaire dans la phase élastique et obéit au critère de Mohr-Coulomb dans la phase plastique. Ces comportements simplifiés permettent de comparer facilement le comportement homogénéisé avec celui des constituants, et d'en déduire éventuellement des résultats plus généraux.

Les valeurs des caractéristiques considérées pour la matrice et les fractures sont les suivantes:

- Roche cristalline :

Elasticité ; module d'Young $E=72000 \mathrm{MPa}$, coefficient de Poisson $\mathrm{v}=0,25$

Plasticité : cohésion C $=17 \mathrm{MPa}$, angle de frottement interne $\phi=57^{\circ}$

- Fractures naturelles colmatées avec de la calcite : Élasticité : raideur normale $\mathrm{k}_{\mathrm{n}}=4.10^{5} \mathrm{MPa} / \mathrm{m}$, raideur tangentielle $\mathrm{k}_{\mathrm{i}}=3.10^{6} \mathrm{MPa} / \mathrm{m}$

Plasticité : cohésion $\mathrm{c}=1,5 \mathrm{MPa}$, angle de frottement interne $\varphi=27^{\circ}$

\section{3}

\section{Méthode numérique}

Considérons le cas d'un solide avec des fractures (Fig. 1). On impose à la frontière de ce solide des dépla- 


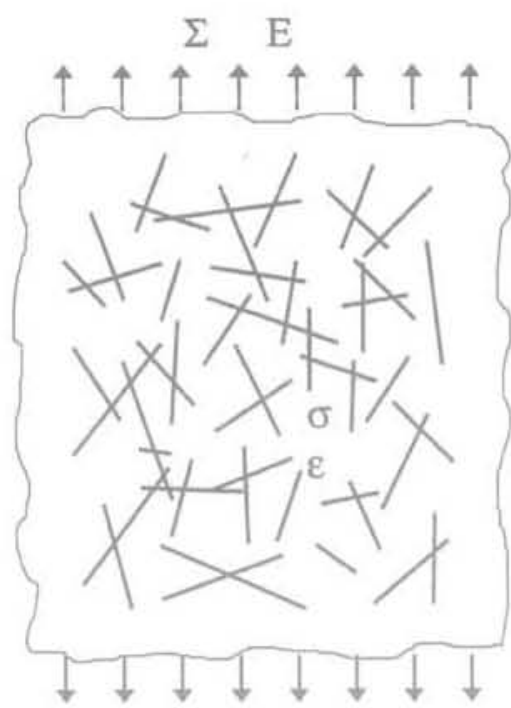

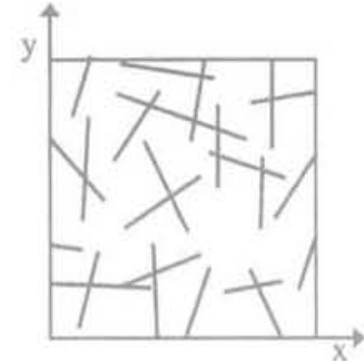

Volume Elémentaire Représentatif

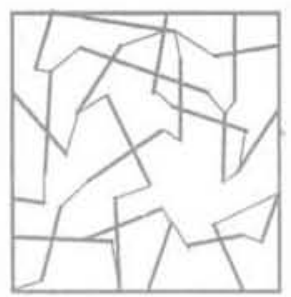

Division du VER en sous domaines fermés

FlG.1 Principe de la méthode numérique développée ; division du VER en sous-domaines fermés. Numerical method: subdivision of the Representative Elementary Volume (REV) in closed subdomains.

cements U correspondant à des déformations macro-

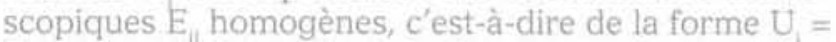

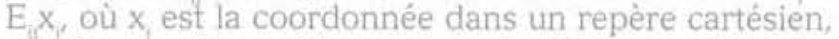
ou bien des forces $\mathrm{Fi}$ correspondant à des contraintes macroscopiques $\Sigma$ homogènes, c'est-à-dire de la forme $\mathrm{F}_{1}=\Sigma \mathrm{n}$, où n est la normale extérieure à la frontière du domaine au point considéré. On suppose que ce chargement crée dans le solide à l'échelle locale (celle des hétérogénéités), des contraintes et déformations désignées par $\sigma$ et $\varepsilon$. On note respectivement $\Sigma^{\text {hom }}$ et $E^{\text {trom }}$ la moyenne (volumique) de $\sigma$ et de $\varepsilon$ dans le domaine. Notre objectif est d'établir un " modèle de comportement homogénéisé $n$ reliant $\Sigma^{\text {hom }}$ à Ehom. Dans le cas où des déplacements $U_{i}$ (resp. forces F) correspondant à une déformation homogène $\mathrm{E}$ (resp. contrainte homogène $\Sigma$ ) sont imposés à la frontière du modèle, on peut montrer que $E^{\text {hom }}=E$ (resp. $\Sigma^{\text {hom }}=\Sigma$ ).

Les quantités moyennes $\Sigma^{\mathrm{h} o m}$ à Enam sont calculées sur un volume suffisamment grand (VER) pour être à l'abri des fluctuations statistiques et représentatives du MHE. La taille du VER est déterminée par des calculs mécaniques préliminaires, à partir de la méthode des éléments finis, sur des domaines de tailles différentes : le VER correspond à une certaine taille critique au-delà de laquelle la valeur d'un paramètre de référence, par exemple le module de Young, ne varie plus avec la taille du domaine. Pour l'exemple étudié ici, la taille du VER est d'environ $10 \mathrm{~m}$. Le VER correspond donc à un carré (distribution uniforme des fractures) de $10 \mathrm{~m}$ de côté (Fig. 1).

Dans la pratique, le calcul aux éléments finis nécessite une discrétisation du domaine à partir d'un maillage respectant la géométrie des fractures. Les fractures (les joints) ne doivent pas traverser les éléments finis (la matrice) du maillage. Pour satisfaire cette exigence, nous avons dû développer un logiciel permettant de diviser automatiquement le VER en sousdomaines fermés. Les fractures en constituent les frontières (Fig. 1). Le domaine complet formé par les sous-domaines fermés ainsi créés peut alors être discrétisé à l'aide d'un préprocesseur du maillage habi- tuel. La dernière étape précédant le calcul consiste à dédoubler les ncuds correspondant aux fractures, pour former les éléments « joints ».

Le calcul est effectué en contraintes planes; cette hypothèse facilite la détermination du comportement homogénéisé dans le plan considéré.

\section{3}

\section{Chargements étudiés et résultats obtenus} VER

Trois types de chargements ont été imposés au

1) Compression dans la direction $x$ avec différentes valeurs de la contrainte latérale $\sigma_{\mathrm{vy}}$ :

$U_{x}$ imposé sur les côtés parallèles à $y, \sigma_{y y}$ constante sur les côtés parallèles à $\mathrm{x}$;

2) Compression dans la direction y avec différentes valeurs de la contrainte latérale $\sigma_{\mathrm{xx}}$

$U_{v}$ imposé sur les côtés parallèles à $x, \sigma_{x x}$ constante sur les côtés parallèles à $y$ :

3) Cisaillement XY avec différentes valeurs de la contrainte moyenne :

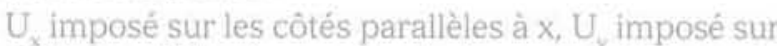
les côtés parallèles à $\mathrm{y}, \sigma$ constante sur les côtés parallèles à $x, \sigma_{y y}=\sigma_{x x}$ constante sur les côtés parallèles à $y$.

Dans chaque cas, les contraintes et les déformations homogénéisées sont calculées à partir de valeurs des forces ou des déplacements imposées ou obtenues sur la frontière. Les courbes de contrainte-déformation issues de ces trois types de chargement sont présentées sur la figure 2. Pour simplifier l'écriture, sont notées dans la suite $\sigma$ et $\varepsilon$ respectivement les contraintes et les déformations homogénéisées. C'est l'analyse de ces résultats qui permet de déterminer le modèle du comportement du MHE. 


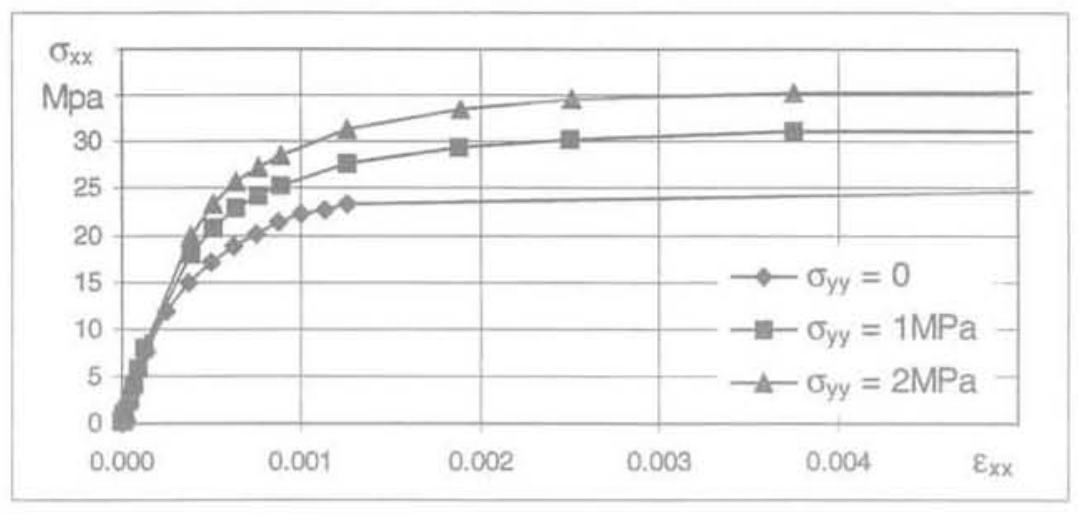
relatives aux différentes directions de chargement.

Stress-strain curves for a homogenized fractured rock mass corresponding to different load directions.
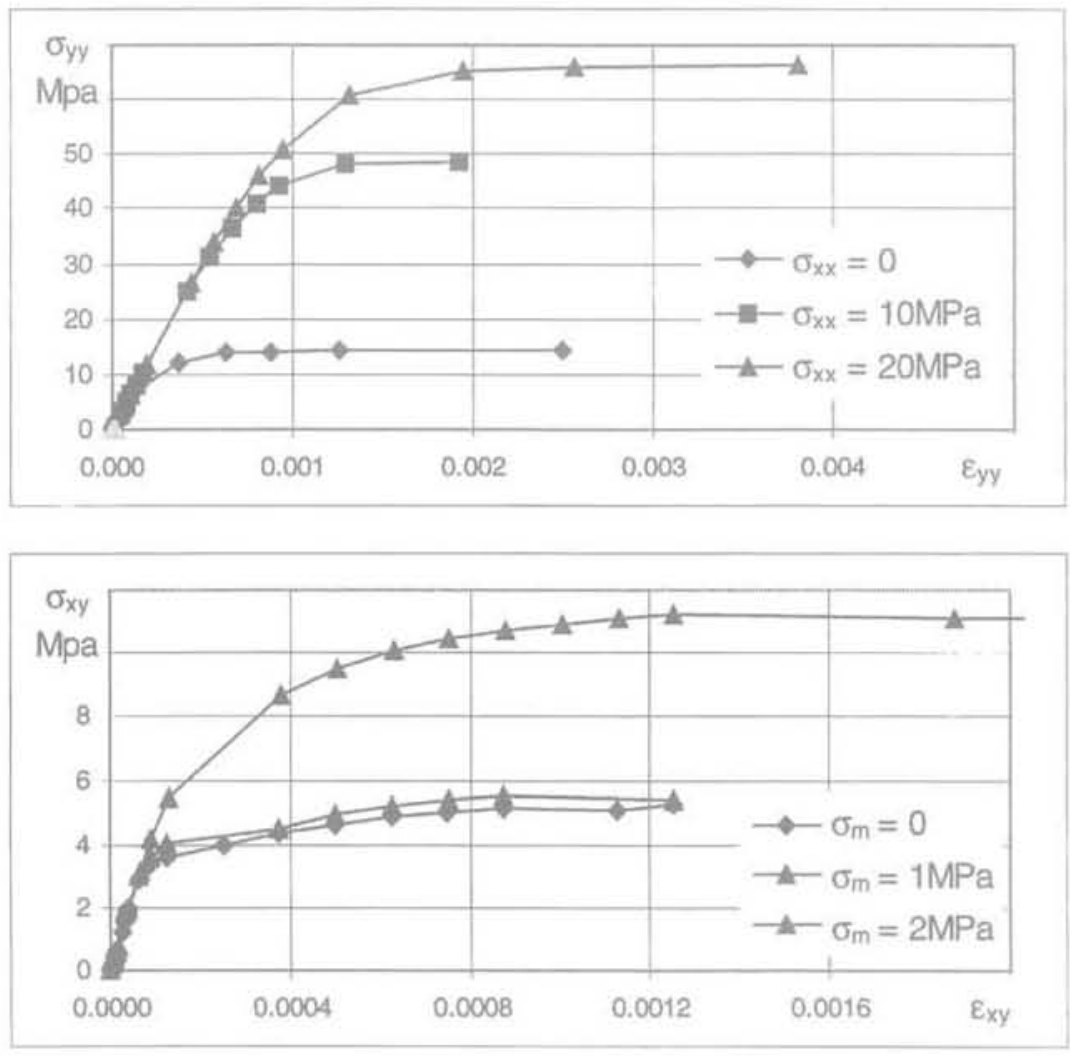

\section{Analyse des résultats}

Les résultats ci-dessus peuvent être assimilés aux "données expérimentales » qui seraient obtenues lors d'une expérience de laboratoire, en vue de l'élaboration d'un modèle rhéologique. On se retrouve confronté aux problèmes habituels de la détermination d'un modèle à partir de données disponibles : celles-ci ne suffisent pas, à elles seules, à la mise au point du modèle et doivent être complétées par des hypothèses sur la forme de la loi de comportement et sur la nature des critères de résistance. Cela peut apparaître surprenant dans la mesure où la simulation numérique permet a priori d'imposer tout trajet de chargement dans toute direction souhaitée, ce qui signifie qu'il est en principe possible de déterminer rigoureusement le modèle de comportement sans aucune hypothèse supplémentaire. Il n'en demeure pas moins qu'en dépit du progrès impor- tant des moyens de calcul les simulations numériques nécessaires à cette étude sont encore longues et fastidieuses (plusieurs jours de calcul sur une station SUN Solaris pour une seule simulation) et peuvent difficilement être répétées en grand nombre. C'est pourquoi nous allons nous contenter de quelques hypothèses complémentaires permettant de pousser plus loin l'interprétation des résultats obtenus. Le bien-fondé des hypothèses adoptées pourrait faire l'objet d'une vérification ultérieure.

Cette démarche conduit à proposer un modèle homogénéisé caractérisé volontairement par des expressions simples qui impliquent des approximations sur l'évolution des résultats obtenus. En revanche, certains aspects du comportement sont déterminés rigoureusement par la voie analytique, à partir des propriétés des différents constituants. C'est le cas par exemple de la limite d'élasticité initiale du MHE, comme nous le verrons dans la suite. 


\section{Modélisation rhéologique}

Trois phases de comportement distinctes sont visibles sur les courbes " contrainte-déformation » présentées ci-dessus : l'élasticité, la plasticité avec écrouissage et la plasticité parfaite. Il convient de souligner que le MHE présente un écrouissage bien que le comportement des constituants du milieu soit parfaitement plastique et ne soit donc caractérisé par aucun écrouissage. Ce fait traduit un effet de structure du milieu hétérogène. Ce phénomène est également rencontré pour d'autres types de milieux hétérogènes tels que les polycristaux (Pouya et al., 1996).

Dans la suite, seront traitées d'abord les deux phases élastique et parfaitement plastique qui sont plus simples à modéliser. La phase avec écrouissage qui fait la transition entre les deux autres sera étudiée à la fin.

\section{1}

\section{Phase élastique}

Les courbes $\alpha$ contrainte-déformation n commencent par une phase élastique linéaire s'étendant jusqu'à des déformations de l'ordre de $10^{-5}$ (la limite d'élasticité du massif sera analysée plus loin).

Le tableau I présente les rapports constants entre les différentes variables caractérisant cette phase à partir des courbes de compression simple et de cisaillement pur.

TABLEAU1 Rapports des différentes variables, obtenus dans la phase élastique linéaire.

\begin{tabular}{|c|c|c|c|}
\hline Direction de chargement & $\sigma_{\mathrm{x} x} / \varepsilon_{\mathrm{n}}(\mathrm{GPa})$ & $\varepsilon_{5 y} / \varepsilon_{\mathrm{xi}}$ & $\varepsilon_{x y} / \varepsilon_{\mathrm{so}}$ \\
\hline$x X$ & 63,2 & 0,269 & 0,015 \\
\hline Direction de chargement & $\sigma_{y} / \varepsilon_{y y}(\mathrm{GPa})$ & $\varepsilon_{x} / \varepsilon_{y y}$ & $\varepsilon_{x p} / \varepsilon_{y y}$ \\
\hline YY & 62,0 & 0,264 & 0,005 \\
\hline Direction de chargement & $\sigma_{x /} / \varepsilon_{x y}(\mathrm{GPa})$ & $\varepsilon_{x} / \varepsilon_{x y}$ & $\varepsilon_{5 y} / \varepsilon_{\mathrm{n}, \mathrm{r}}$ \\
\hline$X Y$ & 49,4 & $-0,014$ & $-0,03$ \\
\hline
\end{tabular}

On remarque que tout chargement dans la direction $X X$ ou suivant $Y Y$ entraine des déformations $\varepsilon_{x y}$ très faibles, voire théoriquement nulles si le matériau homogénéisé est isotrope ou si les directions $\mathrm{X}$ et $\mathrm{Y}$ sont les directions principales de l'anisotropie. Les valeurs de $\varepsilon_{x y} / \varepsilon_{x x}$ et $\varepsilon_{x} / \varepsilon_{y y}$ restent d'ailleurs très faibles, négligeables devant celles du ratio $\varepsilon_{\mathrm{v}} / \varepsilon_{\mathrm{xx}}$ définissant le coefficient de Poisson. En outre, les valeurs des modules d'élasticité obtenues dans les deux directions $\mathrm{XX}$ et $\mathrm{YY}$ ne diffèrent pas significativement. Le comportement élastique peut donc être considéré, en première approximation, comme isotrope. Ce résultat va en faveur d'une hypothèse du comportement élastique isotrope et permet d'accéder aux valeurs des caractéristiques élastiques homogénéisées du massif, données ci-dessous :

$$
\begin{array}{ll}
\text { Module d'Young: } & E=62,6 \mathrm{GPa} \\
\text { Coefficient de Poisson : } & \mathrm{v}=0,267
\end{array}
$$

Ces résultats conduisent à une valeur du module de cisaillement $\mathrm{G}=\mathrm{E} / 2(1+v)=24,7 \mathrm{GPa}$. C'est précisément la valeur obtenue à partir d'une sollicitation en cisaillement (troisième ligne du tableau ci-dessus, où l'on trouve $G=\sigma_{x y} / 2 \varepsilon_{x y}=24,7 \mathrm{GPa}$ ). Cette vérification confirme la cohérence des résultats et la précision des calculs.

\section{2}

\section{Limite de la phase élastique}

La détermination de la limite du domaine d'élasticité initial est importante, car elle indique le début de l'endommagement du massif. Cette limite peut être calculée rigoureusement à l'aide d'un calcul analytique utilisant la théorie du calcul à la rupture. Le domaine d'élasticité du milieu homogénéisé est l'intersection des domaines d'élasticité de ses constituants. Comme dans le cas étudié, la cohésion $\mathrm{C}$ de la matrice est très grande devant celle des fractures c (respectivement 17 et $1,51 \mathrm{MPa}$ ), ce sont les fractures qui atteignent, les premières, la plasticité et qui délimitent, comme nous allons le voir, le domaine d'élasticité du massif.

Considérons une fracture faisant un angle $\theta$ avec la direction $x$. Soient $\sigma_{x x} \sigma_{y y}$ et $\sigma_{x y}$ les composantes du tenseur des contraintes $\sigma \cdot \overrightarrow{\mathrm{t}}=(\cos \theta, \sin \theta)$ et $\overrightarrow{\mathrm{n}}=(-\sin \theta$, $\cos \theta)$ sont les vecteurs respectivement tangent et normal à la fracture. La contrainte de cisaillement $\tau$ dans la direction $\vec{t}$ et la contrainte normale $\sigma_{n}$ sur la fracture sont données par:

$$
\begin{aligned}
& \sigma_{\mathrm{n}}=\overrightarrow{\mathrm{n}} \cdot \sigma \cdot \overrightarrow{\mathrm{n}}=\sigma_{\mathrm{m}}+\mathrm{s} \cos 2 \theta-\sigma_{\mathrm{xy}} \sin 2 \theta \\
& \sigma_{\mathrm{m}}=\frac{1}{2}\left(\sigma_{\mathrm{xx}}+\sigma_{\mathrm{yy}}\right) \\
& \tau=\overrightarrow{\mathrm{t}} \cdot \sigma \cdot \overrightarrow{\mathrm{n}}=-\mathrm{s} \sin 2 \theta+\sigma_{\mathrm{xy}} \cos 2 \theta \\
& \mathrm{s}=\frac{1}{2}\left(\sigma_{\mathrm{xx}}-\sigma_{\mathrm{yy}}\right)
\end{aligned}
$$

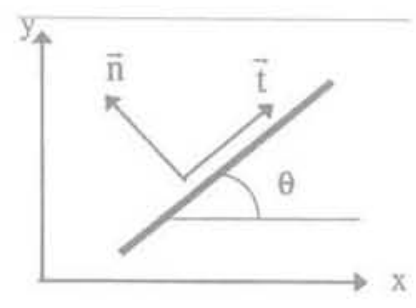

\section{FIG.3 Échelle locale d'une fracture. Local scale for a fracture.}

Le domaine d'élasticité de la fracture est défini par l'inégalité suivante (la contrainte de compression est négative) :

$$
|\tau|<c-\sigma_{n} \operatorname{tg} \varphi
$$

En nous plaçant dans le plan (s, $\left.\sigma_{x y}\right)$, et en notant dans ce plan $S=\left(s, \sigma_{x y}\right), U=(\cos 2 \theta, \sin 2 \theta)$ et $\mathrm{V}=$ $(-\sin 2 \theta, \cos 2 \theta)$, la condition (1) s'écrit :

$$
|\mathrm{S} . \mathrm{V}|<\mathrm{c}-\sigma_{\mathrm{m}} \operatorname{tg} \varphi+\mathrm{S} . \mathrm{U} \operatorname{tg} \varphi
$$


Cette condition définit un domaine compris entre deux droites $D_{1}$ et $D_{2}$ faisant un angle $\varphi$ avec la direction U (Fig. 4). Le dómaine élastique du système des fractures est l'intersection des domaines ainsi obtenus pour différentes valeurs de $\theta$. Lorsque l'angle $\theta$ varie dans un intervalle $\left[\theta_{2}, \theta_{2}\right]$, ce domaine est délimité par

des arcs de cercles et des segments de droites. Ce domaine peut être ouvert ou fermé suivant les valeurs de $\theta_{1}, \theta_{2}$. Pour le massif étudié, $\theta$ varie entre 0 et 90 . De ce fait, Fintersection des domaines élémentaires est un cercle de centre $O$ et de rayon $R$ donné par:

$$
\mathrm{R}=|\mathrm{OA}| \sin \varphi
$$

Soit :

$$
R=c \cos \varphi-\sigma_{m} \sin \varphi
$$

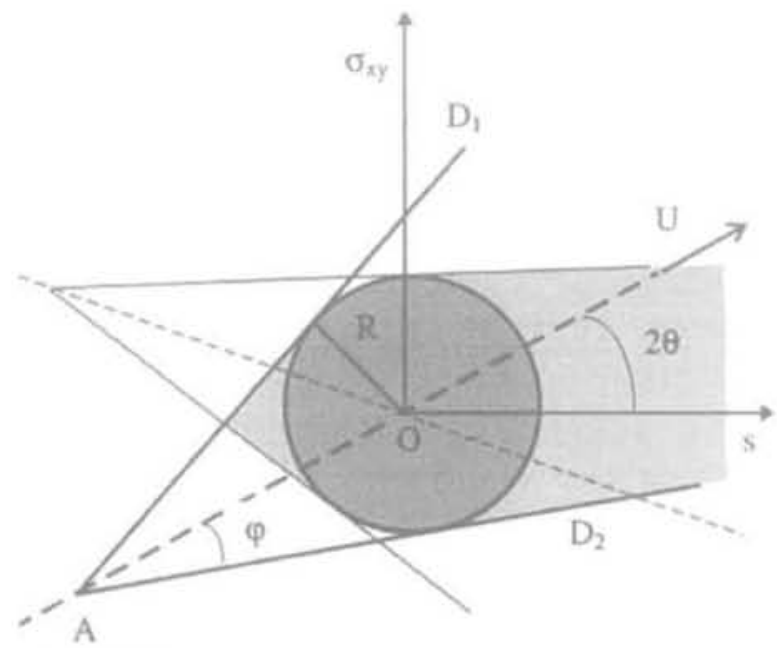

FG.4 Domaine d'élasticité initial du massif fracturé dans une vision homogénéisée. Initial elastic domain of the homogenized rock mass.

Le domaine élastique du massif est l'intersection de ce domaine avec celui de la matrice. Ce dernier correspond à un cercle de centre $\mathrm{O}$ avec un rayon donné par la même expression (2) mais caractérisée par $C$ et $\phi$ de la matrice; le rayon correspondant est donc plus grand que R. En définitif, le domaine élastique du massif est caractérisé par le cercle de rayon $\mathrm{R}$, tandis que le critère de plasticité initial s'écrit:

$$
\sigma_{x y}^{2}+s^{2}<R^{2}
$$

Cette inégalité peut être exprimée sous la forme:

$$
\begin{aligned}
& \sqrt{\sigma_{x y}^{2}+\left(\frac{\sigma_{x x}-\sigma_{y y}}{2}\right)^{2}}<R=c \cos \varphi \\
& -\frac{\sigma_{x x}+\sigma_{y y}}{2} \sigma_{m} \sin \varphi
\end{aligned}
$$

On y reconnait l'expression du critère de MohrCoulomb de cohésion c et d'angle de frottement interne $\varphi$.

En conclusion, le domaine élastique initial du massif fracturé homogénéisé est isotrope et déterminé uniquement par le comportement des fractures.

\section{Phase de plasticité parfaite - critère de résistance}

Comme le montre la figure 2, la courbe d'écrouissage tend vers une asymptote horizontale exprimant une plasticité parfaite, La contrainte asymptotique représente la résistance maximale du matériau dans la direction de chargement considérée. Les valeurs de cette contrainte en fonction de la contrainte latérale ou de la contrainte moyenne sont données dans le tableau II.

TABLEAU il Valeurs de la résistance dans différentes directions en fonction des contraintes latérales ou moyennes.

\begin{tabular}{c|c|c|c}
\hline \multicolumn{1}{c|}{ Direction XX } & & & \\
\hline Contrainte latérale $\sigma_{\mathrm{yy}}(\mathrm{MPa})$ & 0 & 1 & 2 \\
\hline Résistance $\sigma_{\mathrm{sx}}(\mathrm{MPa})$ & 26,0 & 31,1 & 35,4 \\
\hline Direction $\mathrm{YY}$ & & & \\
\hline Contrainte latérale $\sigma_{\mathrm{x}}(\mathrm{MPa})$ & 0 & 10 & 15 \\
\hline Résistance $\sigma_{\mathrm{yy}}(\mathrm{MPa})$ & 14,2 & 49,0 & 66,5 \\
\hline Direction XY & & & \\
\hline Contrainte moyenne $\sigma_{\mathrm{m}}(\mathrm{MPa})$ & 0 & 1 & 10 \\
\hline Résistance $\sigma_{\mathrm{xy}}(\mathrm{MPa})$ & 5,1 & 5,55 & 11,2 \\
\hline
\end{tabular}

Les résultats rassemblés dans le tableau II mettent en évidence une forte anisotropie du comportement plastique, liée aux écarts entre les valeurs de la résistance à la compression uniaxiale relatives aux directions $x$ et $y$. C'est un aspect important du comportement mécanique qui doit être exprimé par le modèle du comportement plastique. Les résultats montrent également une dépendance linéaire de la résistance maximale correspondant aux différentes directions en fonction de la contrainte moyenne ou de la contrainte latérale (Fig. 5).

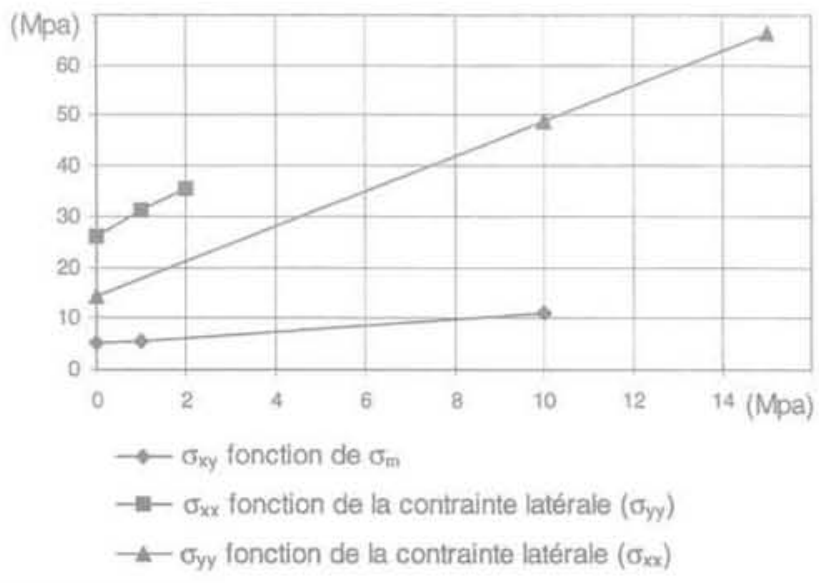

FG.5 Effet de la contrainte normale sur la résistance en cisaillement et de la contrainte latérale sur la résistance en compression simple dans les directions $x$ et $y$.

Effect of lateral or of mean stress on the shear or the compressive strength in different directions. 
Ces résultats permettent de calculer les valeurs de l'angle de frottement interne et de la cohésion pour le milieu homogénéisé. Le tableau III présente ces valeurs obtenues à partir d'une analyse basée sur le chargement dans les directions XX, YY et XY.

TABLEAU III Valeurs de la cohésion et de langle de frottement interne homogénéisés calculées pour les différentes directions de chargement.

\begin{tabular}{|c|c|c|}
\hline Direction de chargement & Coum (MPa) & $\theta^{\text {hina }}$ \\
\hline $\mathrm{XX}$ & 6,02 & $40.5^{2}$ \\
\hline YY & 3,35 & $39,4=$ \\
\hline$X Y$ & 5,02 & $31,7=$ \\
\hline
\end{tabular}

Il convient de remarquer que les valeurs de la cohésion et de l'angle de frottement interne homogénéisés se situent bien, comme cela doit ètre le cas, entre les valeurs des fractures $\left(c=1,51 \mathrm{MPa}, \varphi=27^{\circ}\right)$ et les caractéristiques de la matrice rocheuse $\left(\mathrm{C}=17 \mathrm{MPa}, \phi=57^{\circ}\right)$.

A partir de ces données, le critèré de résistance maximale correspondant à la phase de plasticité parfaite du MHE a été modélisé moyennant quelques hypothèses sur la forme de ce domaine et des approximations sur les valeurs des paramètres.

Puisque les valeurs de l'angle de frottement trouvées dans les directions X et $\mathrm{Y}$ sont très proches, elles seront désormais considérées comme identiques et caractérisées par la valeur moyenne de $40^{\circ}$. En revanche, les valeurs de la cohésion dans les deux directions demeureront bien distinctes.

Supposons maintenant que $\sigma_{4}=0$ et plaçons-nous dans le plan $(\sigma$, s) (Fig. 6). Les deux valeurs de la cohésion données dans le tableau III et la valeur de l'angle de $\pm 40^{\circ}$ définissent deux droites dans ce plan délimitant le domaine de résistance du milieu (noter que changer $\mathrm{X}$ en $\mathrm{Y}$ revient à changer $\mathrm{s}$ en $-\mathrm{s}$ et donc $\varphi$ et $c$ $e n-\varphi$ et $-c)$. Ces droites passent par les points $B=(0$, $6,02)$ et $C(0,-3,35)$ et se croisent au point $A(5,59 ; 1,335)$. Ainsi est obtenue la coupe du domaine de résistance dans le plan $\left(\sigma_{m}\right.$, s)

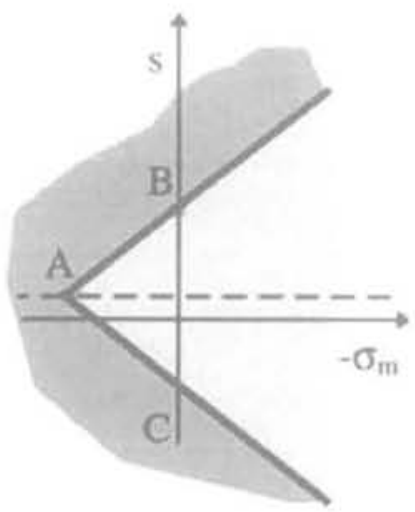

FlG. 6 Le domaine de résistance dans le plan $\left(\sigma_{\mathrm{m}}\right.$ s) est délimité par deux droites de pente \pm $40^{\circ}$ par rapport à l'axe $-\sigma_{\text {. }}$.

The strength domain in $\left(\sigma_{m}, \mathrm{~s}\right)$ plane is bounded by two straight lines making an angle $\pm 40^{\circ}$ with $\sigma_{\mathrm{m}}$ axis.
Fixons maintenant la valeur de $\sigma$ et plaçons nous dans le plan $\left(\mathrm{s}, \sigma_{\mathrm{x}}\right.$ ) passant par cette valeur de $\sigma_{\mathrm{m}}$ (Fig. 7 ). Nous pouvons alors identifier dans le plan $\left(s, \sigma_{w}\right)$, trois points appartenant à la frontière du domaine dé résistance du milieu : deux points dont les coordonnées sont données par $\sigma_{\mathrm{vy}}=0$ et par les valeurs de s exprimées par les deux droites ci-dessus et un point $\mathrm{s}=0$ avec $\sigma_{x y}=5,02$ - $\sigma_{\operatorname{mg}}\left(31,7^{\circ}\right)$. Nous faisons alors l'hypothèse que le domaine de résistance est une ellipse dans ce plan passant par ces points, d'axes parallèles aux axes s et $\sigma$ et centrée sur la même ordonnée s que le point A de la figure 6 . A l'intérieur de ce domaine, on retrouve le domaine d'élasticité initial de MHE (cercle centré sur l'origine) pour la valeur de $\sigma_{m}$ considérée.

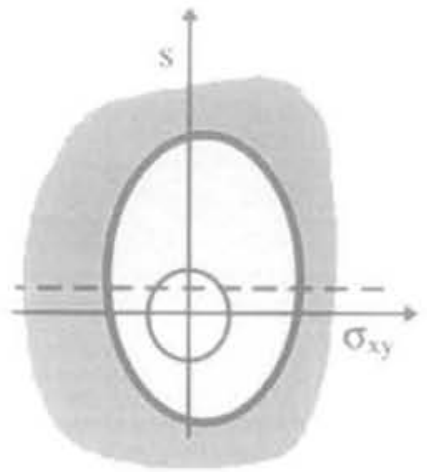

HG.7 Le domaine de résistance dans le plan $\left(\sigma_{\text {, }}\right.$ s) est une ellipse contenant le domaine d'élasticité initial (cercle centré sur lorigine).

The strength domain in $\left(\sigma_{w}\right.$ s) plane is an ellipse containing the initial elastic domain.

La forme elliptique supposẻe pour le domaine de résistance dans le plan $(\sigma$, s) et la variation linéaire des axes de cette ellipse avec la contrainte moyenne conduisent à rechercher le critère de résistance du MHE sous la forme:

$f(\sigma)=f\left(\sigma_{m} s, \sigma_{x y}\right)=(a s-d)^{2}+\left(b \sigma_{x y}-d\right)^{2}-\left(-\sigma_{m}+g\right)^{2}-$ $\mathrm{d}^{2} \leq 0$

où $\mathrm{a}, \mathrm{b}$, d et g sont quatre constantes à déterminer.

En posant $\sigma_{x y}=0$, on trouve : $f\left(\sigma_{m}, \mathrm{~s}, 0\right)=(\mathrm{a} \mathrm{s}-\mathrm{d})^{2}-$ $\left(-\sigma_{m}+g\right)^{2}=0$, ce qui permet de déterminer, d'après la figure 6 et les valeurs du tableau (3) : $\mathrm{a}=\operatorname{cotg}\left(40^{\circ}\right), \mathrm{d}=$ $1,59 \mathrm{MPa}, \mathrm{g}=5,59 \mathrm{MPa}$.

En posant $s=0$, on trouve : $f\left(\sigma_{m}, 0, \sigma_{x p}\right)=\left(b \sigma_{x y}-d\right)^{2}-$ $\left(-\sigma_{+}+g\right)^{2}=0$, ce qui permet d'obtenir : $b=\operatorname{cotg}\left(31,7^{\circ}\right)$. Cette expression conduit à deux droites exprimant la variation de $\sigma_{\mathrm{zy}}$ en fonction de $\sigma_{\mathrm{m}}$ et s'écrivant respectivement: $\begin{aligned} \sigma_{x y} & =-\sigma_{m} \operatorname{tg}\left(31,7^{\circ}\right)+4,43 \mathrm{MPa} \text { et }-\sigma_{x y}=-\sigma_{\mathrm{m}} \operatorname{tg}\left(31,7^{\circ}\right) \\ -2,47 \mathrm{MPa} & \end{aligned}$

La valeur de la cohésion c intervenant dans la première expression est de 4,43 MPa au lieu de 5,02, valeur donnée dans le tableau III. C'est moyennant cette approximation que le critère de résistance est exprimé sous une forme simple donnée par (3). Ce modèle prévoit, par ailleurs, une limite de cisaillement de 2,47 MPa dans la direction opposée à celle étudiée. Ce point est à vérifier par des calculs complémentaires. 


\section{Modèle d'écrouissage}

Le domaine d'élasticité initial du MHE, cercle centré sur l'origine et de rayon $\mathrm{R}$ exprimé par (2), peut se mettre également sous la forme (3). Les valeurs des paramètres correspondants seront alors les suivantes:

$$
\begin{aligned}
\mathrm{a}=\mathrm{b}=1 / \sin \left(27^{\circ}\right)=2,20 \quad \mathrm{~d}=0 \quad \mathrm{~g}=1,51 \operatorname{cotg}\left(27^{\circ}\right) \\
=2,96 \mathrm{MPa} .
\end{aligned}
$$

Nous pouvons alors supposer que le domaine d'élasticité du MHE est défini systématiquement par un critère de la forme (3) dont les parametres a, b, d, g dépendent de l'état du système. Nous supposons plus précisément que ces paramètres dépendent d'une seule variable d'écrouissage, notée $\xi$, déterminée par la déformation plastique du milieu à partir de la relation

\section{$\xi=\mid \hat{e} \|$ avec une valeur initiale nulle. Notons alors $a_{0}$}

$\mathrm{b}_{0}, \mathrm{~d}_{0}, \mathrm{~g}_{0}$ et $\mathrm{a}_{-}, \mathrm{b}_{-}, \mathrm{d}_{-}, \mathrm{g}_{-}$respectivement les valeurs initiales et asymptotiques de a, b, d et g correspondant à la frontière élastique initiale et au critère de résistance maximale. Supposons en plus que la dépendance de ces paramètres en fonction de ş $^{\prime}$ 'exprime sous la forme :

$$
\mathrm{a}=\mathrm{a}_{0}+\left(\mathrm{a}_{-}-\mathrm{a}_{0}\right)\left(1-\mathrm{e}^{-\beta^{3}}\right)
$$

avec des formes analogues pour b, d, et $g$.

Le paramètre $\beta$ est une constante à déterminer par l'ajustement des courbes d'écrouissage. Ce traitement conduit aux valeurs comprises entre 547 et 2447 . Pour simplifier, une valeur unique de $\beta=1000$ sera retenue pour tous les paramètres a, b, d et g. Ce choix est motivé par les résultats présentés sur la figure 8. Il s'agit de la courbe d'écrouissage dans la direction y. L'ajustement de cette courbe conduit à une valeur de $\beta$ $=2447$. La comparaison de la courbe d'écrouissage caractérisée par cette valeur et celle obtenue avec une valeur de $\beta=1000$ nous autorisent à restreindre le nombre de paramètres du modèle, ce qui revient à considérer que l'écart observé sur la figure 8 est acceptable, compte tenu des incertitudes pesant sur la connaissance des caractéristiques d'un milieu fracturé.

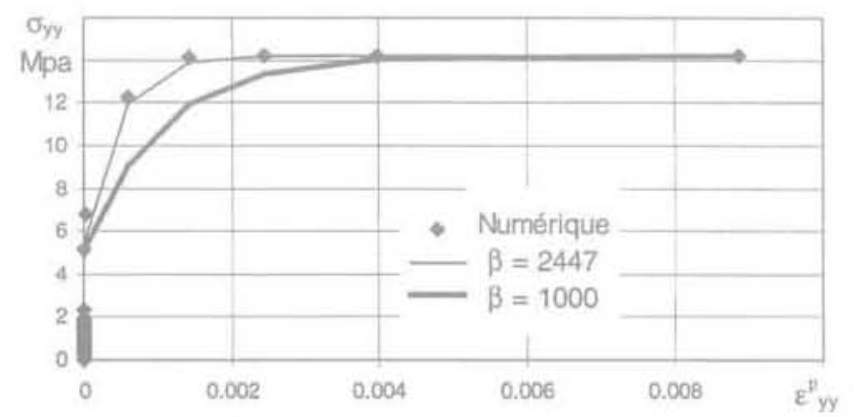

FG. 8 Comparaison entre Yajustement individuel $(\beta=2447)$ de la courbe d'écrouissage dans la direction y et l'ajustement avec une valeur de $\beta$ unique $(\beta=1000)$.

Comparison between the numerical plot (corresponding to compression in y direction), the individual fitting of this plot, leading to $\beta=2,447$, and fitting of this plot with a fixed value of $\beta=1,000$.
En résumé, le comportement plastique du MHE est décrit comme suit:

Critère de plasticité :

$f(\sigma, \xi)=f\left(\sigma_{m,}, s, \sigma_{x y}, \xi\right)=(\mathrm{a} \mathrm{s}-\mathrm{d})^{2}+\left(\mathrm{b} \sigma_{x y}-\mathrm{d}\right)^{2}-(-) \sigma_{m}$
$+\mathrm{g})^{2}-\mathrm{d}^{2} \leq 0$

Loi d'écrouissage :

$$
\begin{aligned}
& \dot{a}=\beta\left(a_{-}-a_{0}\right) e^{-k} \xi \\
& \dot{b}=\beta\left(b_{-}-b_{f}\right) e^{*} \xi \\
& \dot{d}=\beta\left(d_{-}-d_{1}\right) e^{* k} \xi \\
& \dot{g}=\beta(g-g) e^{* n} \xi
\end{aligned}
$$

$$
\dot{\xi}=\|\dot{\varepsilon}-\|
$$

Etat initial: $\quad \xi=0 ; a=a_{0} ; b=b_{0} ; d=d_{0} ; g=g_{0}$ Valeurs numériques : $\beta=1.000$

$$
a_{0}=2,20 ; \quad b_{0}=2,20 ; \quad d_{0}=0 ; g_{0}=2,96 \mathrm{MPa}
$$
$\mathrm{a}_{\mathrm{w}}=1,19 ; \mathrm{b}_{\mathrm{w}}=1,62 ; \mathrm{d}_{\mathrm{w}}=1,59 \mathrm{Mpa} ; \mathrm{g}_{\sim}=5,59 \mathrm{MPa}$

\section{5}

\section{Conclusions et perspectives}

Une méthode numérique a été présentée pour estimer la résistance mécanique de massifs cristallins fracturés. Elle s'appuie sur le relevé de l'état de fracturation du massif et les caractéristiques mécaniques de la matrice rocheuse et des fractures. Son originalité tient à l'approche mise en œuvre qui consiste à assimiler le massif à grande échelle à un milieu homogène équivalent. Les caractéristiques de la déformabilité et de la résistance de ce milieu ont fait l'objet d'une étude fondée sur l'homogénéisation numérique. En mettant à profit les données disponibles sur un massif cristallin, une méthodologie a été mise au point. Elle a permis d'établir un modèle rhéologique susceptible d'être employé ultérieurement pour des études concrètes. La méthode proposée ne prétend pas être exempte de toute hypothèse, simplification ou approximation. Nous pensons cependant que, par rapport aux méthodes empiriques et qualitatives utilisées en mécanique des roches pour les massif́s fracturés, la méthode proposée intègre davantage et plus rigoureusement l'ensemble des informations pouvant être rassemblées, pour ce type de milieu, à partir d'investigations in situ et au laboratoire. Ces données concernent en particulier l'état et les caractéristiques mécaniques des fractures qui jouent un rôle prépondérant dans la résistance du massif rocheux.

Les résuitats obtenus démontrent l'intérêt et la faisabilité de la méthode proposée qui peut être étendue à trois dimensions, sans trop de difficulté. Il semble désormais possible d'établir des abaques à partir de résultats des simulations numériques qui pourraient être réalisées sur quelques configurations typiques de l'état de fracturation des massifs rocheux. Une fois validé par l'expérience de terrain, ce genre d'outil serait très utile à l'étude des massifs fracturés. Il constituerait un complêment indispensable aux outils de modélisation locale employés, par ailleurs, pour l'étude des phénomènes à petite échelle. 


\section{Bibliographie}

Barton N., Lien R., Lunde J. (1974) - « Engineering classification of rock masses for the design of tunnel support ». Rock Mechanics, vol. 6, n० 4. p. $189-236$

Bekaert A. Maghous S. (1996) - $\alpha$ Threedimensional yield strength properties of jointed rock mass as a homogenized medium 11. Mechanics of Cohesive. Frictional Materials, vol. 1, p. 1-24

Bieniawski Z.T. (1976) - « Rock mass clas. sifications in rock engineering 8 . Proceedings of the symposium on Exploration for Rock Engineering, Johannesburg

Buhan (de) P., Maghous S. (1997) - u Com- portement élastique non linéaire macroscopique d'un matériau comportant ur réseau de joints 1. C.R. Acad. Sci. Paris, t. 324, Série II b, p. 209-218.

Coste F. (1997) - « Comportement thermo hydro-mécanique des massifs rocheux fracturés $n$. Thèse de doctorat, École nationale des ponts et chaussees.

Coste F., Ghoreychi M., Didry O. (1999) u Modélisation du comportement hydromécanique des massifs rocheux fracturés par homogénéisation n. Ge Congrès mondial de mécanique des roches, Balkema, p. 875-880.
Long J.C.S., Billaux D. (1987) - « From field data to fracture network modelling : an example incorporating spatial structure n. Water Ressources Research, vol. 23, n 7 , p. 1201-1216.

Pouya M., Zaoul A., Nabouli M. (1996) - «A micro-macro model for polycrystalline halite is. The mechanical behavior of salt Proc $4^{\text {it }}$ Conf., Ghoreychi et al, eds. 'Trans Tech Pub, p. 129-141.

Su K., Ghoreychi M., Chanchole S. (2000) a Experimental study of damage in granite 11 . Geotechnique, vol. L n ${ }^{\circ} 3$, p. 235241. 